Over the last 12 months the DLA have published a free framework, developed training workshops, launched a free hospice inpatient resource, run 'pop-up' events, organised a Digital Legacy conference, carried out different forms of research and campaigned highlighting that end of life and bereavement in relation to the internet and electronic devices is an area requiring thought, governance and change.

The DLA spend a lot of time working with hospices, CCGs, charities and trusts. The outcome is improved knowledge and skillsets applied at an organisational and/or at an individual level. The diffusion of the DLAs information occurs by supporting professionals through a variety of different channels. In turn this helps professionals to better support patients and their families.

The DLA's work often revolves around conversations and campaigning. Their recent work for Velindre NHS Trust, Aneurin Bevan UHB \& Byw Nawr included building a TalkCPR awareness website. The goal of the TalkCPR campaign is to help increase dialogue around CPR and DNACPR within Wales. The TalkCPR campaign was recently nominated for a NHS Wales award.

This paper will document the DLA's journey over the last year, explain how they use creativity to address end-of-life and document how they plan to support every UK hospice over the course of 2017.

\section{P-222 BREAKING TRADITION IN PALLIATIVE CARE- INTRODUCTION OF REMOTE TECHNOLOGY AND VIRTUAL CONSULTATIONS}

Sarah Roberts, Nicola Parkes, Susan Salt, Julie Huttley. Trinity Hospice, Blackpool, UK

\subsection{6/bmjspcare-2016-001245.243}

The aim of the project was to develop a strategic vision in three stages for the palliative care service to improve and expand our reach to patients that incorporated the use of technology. The project aimed to use virtual technology to open up access for consultant led advice in the patients home via video link consultation. This would allow more access to face to face consultations, a more responsive service, less travel time and more access to allow a "tech savvy" population to connect directly with the service.

The second aim of the project was to allow the service to utilise technology to enhance sharing of knowledge and skills to our community colleagues, opening up access to web based educational "bitesize" programmes to improve and enhance skills of our community colleagues.

The final aim of the project is to utilise technology to connect with our community partners to enhance communication and consultation with and about patients. We would use this with our out of hours providers as part of a community hub approach, using technology within the patients home to seek direct clinical advice enhanced by video links.

\section{P-223 PATIENT AND CARER PORTAL}

Nick Middleton, Matt Smith. St Margaret's Hospice, Taunton, UK

\subsection{6/bmjspcare-2016-001245.244}

The Patient and Carer Portal is a multi-layered secure website that is being developed to enable professionals and patients to access our electronic patient records.
Key stakeholders will also be able to access tele-health and educational information and content through the online platform.

Innovative Technology This portal is just one of the ways technology can be used to reach out to more people, and allow us to spread the already stretched funding a little bit further.

Once launched, the platform will continue to grow and help us reach patients in their own homes, where they can receive tele-health services and add notes directly into their own patient record.

Reaching more patients, now and in the future A number of additional features will be released in stages over the next few years, slowly growing the service to reach out to more people and services. This will include the ability to self-refer, and for professionals to refer online 24 hours a day, 7 days a week, 365 days a year.

Tailored to Individuals In addition to providing patients and professionals with remote access to medical records thus allowing them to read notes and directly update the records themselves 'prescribed' information, specifically tailored to the individual patient, will also be available through the Portal.

Educational Content Instructional videos for patients and professionals will also be accessible through the portal. Examples include: therapeutic audio/visual services and content, developed in-house by our highly experienced and professional therapies team (i.e., a self-help video on 'How to deal with breathlessness').

\section{Workforce, Volunteers, Leadership, Education and Workplace}

\section{P-224 CREATION OF A NEW STARTER INFORMATION PACK FOR REGISTERED NURSES IN A HOSPICE IN PATIENT UNIT}

Gemma Hodge, Sharon Allman. St Gemma's Hospice, Leeds, UK

\subsection{6/bmjspcare-2016-001245.245}

'There is still a workforce crisis in the NHS the number one priority for health care providers is making sure they have enough staff to deliver safe care' Davies (2016). This is certainly something we have experienced and as a result in the hospice where previously applications would be from nurses in palliative care we are now employing many nurses from a variety of backgrounds, so their palliative care knowledge can be limited.

Due to this shift in experience, we notice that inducting new starters was more complex than it had been. Entering Palliative care nursing can be very daunting for nurses coming from different specialities, particularly with the doses of medications used 'off licence' (Twycross et al., 2014) that we use to achieve symptom management. We therefore wanted new staff to feel empowered and valued, and felt that through providing them with an information pack when we are encouraging them to think about their knowledge gaps, giving them an information resource and guiding them through that period of induction.

Process of Implementation- What is current in education, relevant e-Learning, introduction to equipment we used, worksheets on key issues in palliative care to engage thinking around topics, face to face sessions on complex issues with advanced nurse practitioner, pharmacist and nurse consultant and requirement to complete a reflection. Feedback was very positive. Inclusion of 